Journal of Electromagnetic Waves and Applications, Vol.11, 1057-1072, 1997

\title{
A COMPARATIVE STUDY OF TWO INTEGRAL-EQUATION FORMULATIONS OF TE MODES IN CIRCULAR RIDGED WAVEGUIDE
}

\author{
S. Amari, J. Bornemann, and R. Vahldieck
}

Laboratory for Lightwave Electronics

Microwaves and Communications (LLiMiC)

Department of Electrical and Computer Engineering

University of Victoria

Victoria B.C. Canada V8W 3P6

\begin{abstract}
We present and compare two integral-equation formulations of the TE eigenvalue problem of a ridged circular waveguide. In the E-formulation, an integral equation for the tangential electric field $E_{\phi}$ is derived and solved by the moment method using basis functions which include the edge conditions. The $\mathrm{H}$-formulation relies on an integral equation for the axial magnetic field $H_{z}$, which is also solved by the moment method and basis functions with edge conditions. Spurious roots are encountered in the E-formulation whereas the H-Formulation is free from spurious roots but requires larger matrices. Results from both formulations are compared with previously published data and analytic results for limiting cases, excellent agreement is demonstrated.
\end{abstract}

\section{INTRODUCTION}

Ridge waveguides are an important component in modern microwave filters, septum polarizers and matching networks. Dual-mode filters using waveguide technology often rely on accurate design and analysis of ridged sections of circular waveguides.

A host of numerical techniques have been employed in the analysis of these structures in the recent years. The cutoff wavelengths of the first two modes of a septum polarizer were determined by Behe and Brachat by the Finite Element Method (FEM) [1]. The Mode-Matching Technique (MMT) was used by Balaji and Vahldieck to investigate the eigenmode problem of single and multiple symmetric ridge waveguides [2]. The Method of Lines (MoL) was also applied to the eigenmode problem of a partially loaded 
ridge waveguide [3]. Ridged structures with 2 symmetry planes in both rectangular and circular waveguides were treated by the Magnetic-FieldIntegral-Equation (MFIE), which was solved using pulses as basis functions, by Sun and Balanis [4].

In this paper, we propose to modify the Mode-Matching Technique (MMT) to allow the inclusion of the edge conditions and eliminate the phenomenon of relative convergence. Instead of relying on the modes of the subregions of the structure, which is the standard practice in MMT, we perform a change of basis functions at the interfaces and introduce an additional degree of freedom in order to always satisfy the boundary conditions of either the electric field or the magnetic field. An integral equation, or a set of coupled integral equations, are then derived for this additional degree of freedom and solved by the moment method.

Attention will be focused only on the Transverse Electric modes since they provide a fertile testing ground for the two integral formulations. In the first formulation, the E-formulation, we derive an integral equation for the quantity $E_{\phi}$ at the interface whereas the H-formulation requires two coupled integral equations for the axial magnetic field $H_{z}$ at the same interfacc. Due the different asymptotic behavior of these two quantities at the edges of the metallic ridge, a sizable disparity in the numerical performance of the two methods is observed. The E-formulation requires smaller matrices than the $\mathrm{H}$-formulation but suffers from the phenomenon of spurious roots which are eliminated when enough basis functions are used.

The paper is organized as follows. Section 2 presents a derivation of the integral equation of $E_{\phi}$ along with a set of basis functions which include the edge conditions. Section 3 discusses the H-formulation and the corresponding basis functions. Scction 4 presents typical numerical results.

\section{A THE E-FORMULATION}

The structure under consideration is shown in figure 1. We assume that all metallic surfaces are perfectly conducting. The ridge of thickness $2 \theta$, and depth $a-b$ has its upper surface at $\phi=0$. We focus our attention first on the TE modes with electric-wall symmetry.

The axial component of the magnetic field, from which the transverse components of the electromagnetic fields of a TE mode are obtained, is expanded in modal series in each of the two regions. The boundary conditions at the metallic surfaces of region II are included in these expansions while 


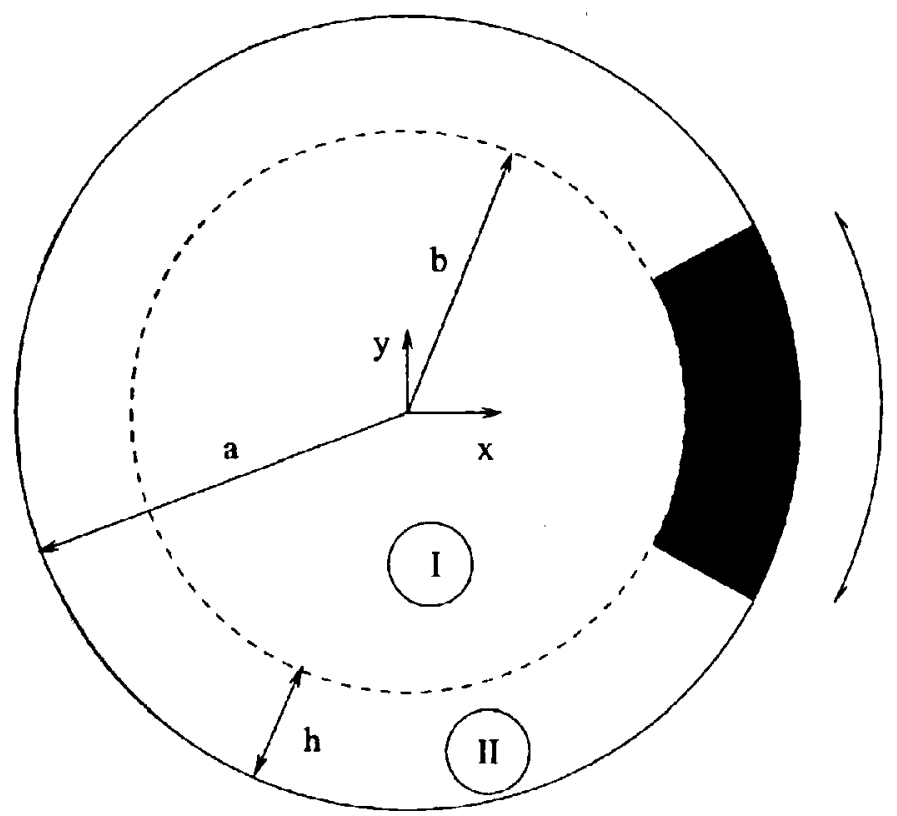

$2 \theta$

Figure 1. Cross section of ridge circular waveguide and its subdivision for modal expansions.

no specific conditions are imposed at the interface I-II.

$$
H_{z}^{I}(\rho, \phi)=\sum_{n=0}^{\infty} J_{n}\left(k_{c} \rho\right) A_{n} \cos (n \phi)
$$

and

$$
H_{z}^{I I}(\rho, \phi)=\sum_{m=0}^{\infty} C_{m}\left[J_{l}^{\prime}\left(k_{c} a\right) Y_{l}\left(k_{c} \rho\right)-Y_{l}^{\prime}\left(k_{c} a\right) J_{l}\left(k_{c} \rho\right)\right] \cos \left[m \pi \frac{\phi-\theta}{\pi-\theta}\right]
$$

where $l=\frac{m \pi}{\pi-\theta}$.

At cutoff, the only non-vanishing components of the electromagnetic field of a TE mode, which are tangential to the interface I-II, are $H_{z}$ and $E_{\phi}$. It is, therefore, sufficient to enforce the boundary conditions of these components which are written as

$$
\begin{gathered}
E_{\phi}^{I}(\rho=b, \phi)=0, \quad-\theta \leq \phi \leq \theta \\
E_{\phi}^{I}(\rho=b, \phi)=E_{\phi}^{I I}(\rho=b, \phi), \quad \theta \leq \phi \leq 2 \pi-0
\end{gathered}
$$


and

$$
H_{z}^{I}(\rho=b, \phi)=H_{z}^{I I}(\rho=b, \phi), \quad \theta \leq \phi \leq 2 \pi-0 .
$$

Instead of following the Mode-Matching Technique (MMT), in which the modal expansions in (1) and (2) are directly substituted in the boundary conditions (3), we introduce an additional degree of freedom in the problem and assume that the true tangential electric field $E_{\phi}$ at the interface is given by an unknown function $X(\phi)$ which vanishes over the metallic part of the interface I-II

$$
X(\phi)=0, \quad-0 \leq \phi \leq \theta,
$$

and rewrite equation (3.b) in the simple yet revealing form

$$
\begin{aligned}
E_{\phi}^{I}(\rho=b, \phi) & =X(\phi) \\
E_{\phi}^{I I}(\rho=b, \phi) & =X(\phi) .
\end{aligned}
$$

It is straightforward to verify that, as long as the function $X(\phi)$ satisfies condition (4), the boundary conditions of $E_{\phi}$ are always satisfied. In addition, whatever information we have on the behavior of $E_{\phi}$ at the intcrface, such as the edge conditions, can be included, through the basis functions, in the function $X(\phi)$.

From equation (5), the modal expansion coefficients in (1) and (2) can be expressed in terms of the function $X(\phi)$.

$$
\begin{aligned}
A_{n} & =\frac{1}{\pi\left(1+\delta_{n 0}\right)} \frac{1}{J_{n}^{\prime}\left(k_{c} b\right)} \int_{\theta}^{2 \pi-\theta} X(\phi) \cos (n \phi) d \phi \\
& =\frac{\tilde{X}^{I c}(n)}{J_{n}^{\prime}\left(k_{c} b\right)}
\end{aligned}
$$

and

$$
\begin{aligned}
C_{m} & =\frac{1}{(\pi-\theta)\left(1+\delta_{m 0}\right)} \frac{\int_{\theta}^{2 \pi-\theta} X(\phi) \cos \left[m \pi \frac{\phi-\theta}{\pi-\theta}\right] d \phi}{J_{l}^{\prime}\left(k_{c} a\right) Y_{l}^{\prime}\left(k_{c} a\right)-Y_{l}^{\prime}\left(k_{c} b\right) J_{l}^{\prime}\left(k_{c} a\right)} \\
& =\frac{\tilde{X}^{I I c}(m)}{J_{l}^{\prime}\left(k_{c} a\right) Y_{l}^{\prime}\left(k_{c} a\right)-Y_{l}^{\prime}\left(k_{c} b\right) J_{l}^{\prime}\left(k_{c} a\right)} .
\end{aligned}
$$

If these expressions of the modal expansion coefficients are used in the continuity condition of $H_{z}$, equation (3.c), we obtain the following integral equation for $X(\phi)$

$$
\begin{aligned}
& \sum_{m=0}^{\infty} \tilde{X}^{I I_{c}}(m) \frac{Y_{l}^{\prime}\left(k_{c} a\right) J_{l}\left(k_{c} b\right)-J_{l}^{\prime}\left(k_{c} a\right) Y_{l}\left(k_{c} b\right)}{Y_{l}^{\prime}\left(k_{c} a\right) J_{l}^{\prime}\left(k_{c} b\right)-J_{l}^{\prime}\left(k_{c} a\right) Y_{l}^{\prime}\left(k_{c} b\right)} \cos \left[m \pi \frac{\phi-\theta}{\pi-\theta}\right] \\
& -\sum_{n=0}^{\infty} \frac{J_{n}\left(k_{c} b\right)}{J_{n}^{\prime}\left(k_{c} b\right)} \tilde{X}^{I c}(n) \cos (n \phi)=0
\end{aligned}
$$


which holds only when $\phi \in[\theta, 2 \pi-\theta]$. The transformations of the function $X(\phi), \tilde{X}^{I c}(n)$ and $\tilde{X}^{I I c}(m)$ are defined in equations $(6)$.

To solve this integral equation, we expand the function $X(\phi)$ in a series of basis functions which satisfy condition (1) along with the edge conditions. Let $B_{i}$ denote a generic element of such a set and write $X(\phi)$ as follows

$$
X(\phi)=\sum_{i=1}^{M} c_{i} B_{i}(\phi)
$$

To determine the expansion coefficients $c_{i}$ 's, we apply Galcrkin's method to the integral equation (7) with the expansion (8) [5]. A set of linear and homogeneous equations in the coefficients $c_{i}$ results

$$
[K][c]=0
$$

where the entries of the matrix $[K]$ are given by

$$
\begin{array}{r}
{[K]_{i j}=\left(1-\frac{\theta}{\pi}\right) \sum_{m=0}^{\infty}\left(1+\delta_{m 0}\right) \tilde{B}_{i}^{I I c}(m) \tilde{B}_{j}^{I I c}(m) \times} \\
\frac{Y_{l}^{\prime}\left(k_{c} a\right) J_{l}\left(k_{c} b\right)-J_{l}^{\prime}\left(k_{c} a\right) Y_{l}\left(k_{c} b\right)}{Y_{l}^{\prime}\left(k_{c} a\right) J_{l}^{\prime}\left(k_{c} b\right)-J_{l}^{\prime}\left(k_{c} a\right) Y_{l}^{\prime}\left(k_{c} b\right)} \\
-\sum_{n=0}^{\infty} \frac{J_{n}\left(k_{c} b\right)}{J_{n}^{\prime}\left(k_{c} b\right)} \bar{B}_{i}^{I c}(n) \tilde{B}_{j}^{I c}(n)\left(1+\delta_{n 0}\right) .
\end{array}
$$

The cutoff wavenumbers are determined as the roots of the determinant of the square and symmetric matrix $[K]$. To avoid the poles in the determinant, the zeros of the smallest singular value of this matrix are located instead [6].

\section{B BASIS FUNCTIONS FOR E-FORMULATION}

The basis functions used to expand $E_{\phi}$ at the interface should capture the salient features of this quantity to guarantee numerical cfliciency. Since $E_{\phi}$ is normal to the axis of the $90^{\circ}$ metallic wedge, it becomes singular as $r^{-1 / 3}$ as the radial distance to the axis of the wedge $r$ approaches zero [7]. Taking into account the fact that there are two wedges located at $\phi=0$ and $\phi=2 \pi-\theta$, a set of basis functions which satisfy these requirements is given by

$$
B_{k}(\phi)=\frac{\cos \left[(k-1) \pi \frac{\phi-\theta}{\pi-\theta}\right]}{[(\phi-\theta)(2 \pi-\theta-\phi)]^{1 / 3}}, \quad k=1,2, \ldots
$$


The spectra of these basis functions in each of the two regions can be expressed in terms of Bessel function of the first kind of order $1 / 6$ [8] $\left(G=0.5 \Gamma\left(\frac{1}{2}\right) \Gamma\left(\frac{2}{3}\right)\right)$

$$
\begin{gathered}
\tilde{B}_{k}^{I c}(n)=\frac{G}{\left(1+\delta_{n 0}\right)}\left\{\frac{J_{1 / 6}[k \pi+n(\pi-\theta)]}{\left|k \frac{\pi}{2}+n \frac{(\pi-\theta)}{2}\right|^{1 / 6}} \cos [(k-1) \pi+n(\pi-\theta)]\right. \\
\left.+\frac{J_{1 / 6}[k \pi-n(\pi-\theta)]}{\left|k \frac{\pi}{2}-n \frac{(\pi-\theta)}{2}\right|^{1 / 6}} \cos [k \pi-n(\pi-\theta)]\right\} \\
\tilde{B}_{k}^{I I c}(m)=\frac{G}{\left(1+\delta_{m 0}\right)}\left\{\frac{J_{1 / 6}[(m-k) \pi]}{\left|(m-k) \frac{\pi}{2}\right|^{1 / 6}} \cos [(m-k) \pi]\right. \\
\left.+\frac{J_{1 / 6}[(m+k) \pi]}{\left|(m+k) \frac{\pi}{2}\right|^{1 / 6}} \cos [(m+k) \pi]\right\}
\end{gathered}
$$

Note that the modes of the two subregions are all included in the theory and rather in a simple way; they appear only in computing the inner products in the quantities $[E]_{i j}$. The phenomenon of relative convergence is eliminated by testing these sums for convergence, thereby leaving only one free parameter in the numerical solution, i.e., the number of basis functions $M$. The spectra of the basis functions of the TE modes with magnetic-wall symmetry can also be expressed in terms of Bessel functions of order $1 / 6$; they are given in Appendix $\mathrm{A}$.

A possible disadvantage of the E-formulation is the fact that a quantity, which is obtained from $H_{z}$ by differentiation, is expanded instead of $I_{z}$ itself. It is, therefore, not surprising that $E_{\phi}$ is infinite at the edges of the ridge whereas $H_{z}$ is finite everywhere [7]. $\Lambda$ lso, $H_{z}$ is not necessarily zero at the metallic edges since a constant is also a possible asymptotic solution at a $90^{\circ}$ wedge. Such a constant is eliminated from the theory when the derivative of $H_{z}$ is taken to obtain $E_{\phi}$. It will be seen that, indecd, this formulation exhibits spurious roots which have not been encountered in the H-formulation.

\section{A THE H-FORMULATION}

In this formulation, we derive an integral equation for the axial magnetic field $H_{z}$ at the interface I-II.

The starting point is again the modal expansions given by equations (1) and (2). The boundary conditions are those in cquations (3). The major difference resides in the fact that the axial magnetic field is non-zero over the entire range $0 \leq \phi \leq 2 \pi$; basis functions which cover this range are 
needed. To accommodate the edge conditions, this interval is split into two parts: a) the gap of the interface $\theta \leq \phi \leq 2 \pi-\theta$ and b) the metallic surface of the ridge $-\theta \leq \phi \leq \theta$. Let $Y(\phi)$ and $Z(\phi)$ denote the true distributions of $H_{z}$ in the first and second intervals, respectivcly, such that

$$
H_{z}^{I}(\rho=b, \phi)=H_{z}^{I I}(\rho=b, \phi)=Y(\phi), \quad 0 \leq \phi \leq 2 \pi-\theta
$$

and

$$
H_{z}^{I}(\rho=b, \phi)=Z(\phi), \quad-\theta \leq \phi \leq \theta
$$

Equations (13) can be combined with equations (1) and (2) to eliminate the modal expansion coefficients leading to

$$
\begin{aligned}
A_{n} & =\frac{1}{\pi\left(1+\delta_{n 0}\right)} \frac{1}{J_{n}\left(k_{c} b\right)} \times\left[\int_{\theta}^{2 \pi-\theta} Y(\phi) \cos (n \phi) d \phi+\int_{-\theta}^{\theta} Z(\phi) \cos (n \phi) d \phi\right] \\
& =\frac{\tilde{Y}^{I c}(n)+\tilde{Z}^{I c}(n)}{J_{n}\left(k_{c} b\right)}
\end{aligned}
$$

and

$$
\begin{aligned}
C_{m} & =\frac{1}{(\pi-\theta)\left(1+\delta_{m 0}\right)} \frac{\int_{\theta}^{2 \pi-\theta} Y(\phi) \cos \left[m \pi \frac{\phi-\theta}{\pi-0}\right] d \phi}{J_{l}\left(k_{c} a\right) Y_{l}^{\prime}\left(k_{c} a\right)-Y_{l}\left(k_{c} b\right) \cdot J_{l}^{\prime}\left(k_{c} a\right)} \\
& =\frac{\tilde{Y}^{I I c}(m)}{J_{l}\left(k_{c} a\right) Y_{l}^{\prime}\left(k_{c} a\right)-Y_{l}\left(k_{c} b\right) J_{l}^{\prime}\left(k_{c} a\right)}
\end{aligned}
$$

Two coupled integral equations for the functions $Y(\phi)$ and $Z(\phi)$ can be derived by using equations (14) in the boundary conditions of $E_{\phi}$ as given in equations (3.a) and (3.b):

$$
\begin{aligned}
& \sum_{n=0}^{\infty} \frac{J_{n}^{\prime}\left(k_{c} b\right)}{J_{n}\left(k_{c} b\right)}\left[\tilde{Y}^{I c}(n)+\tilde{Z}^{I c}(n)\right] \cos (n \phi) \\
- & \sum_{m=0}^{\infty} \frac{J_{l}^{\prime}\left(k_{c} b\right) Y_{l}^{\prime}\left(k_{c} a\right)-J_{l}^{\prime}\left(k_{c} a\right) Y_{l}^{\prime}\left(k_{c} b\right)}{J_{l}\left(k_{c} a\right) Y_{l}^{\prime}\left(k_{c} a\right)-Y_{l}\left(k_{c} b\right) \cdot J_{l}^{\prime}\left(k_{c} a\right)} \tilde{Y}^{I I c}(m) \cos \left[m \pi \frac{\phi-\theta}{\pi-\theta}\right]=0
\end{aligned}
$$

which holds when $\phi \in[0,2 \pi-\theta]$, and

$$
\sum_{n=0}^{\infty} \frac{J_{n}^{\prime}\left(k_{c} b\right)}{J_{n}\left(k_{c} b\right)}\left[\tilde{Y}^{I c}(n)+\tilde{Z}^{I c}(n)\right] \cos (n \phi)=0
$$

which holds when $\phi \in[-\theta, \theta]$. 
To solve these coupled integral equations, we expand the functions $Y(\phi)$ and $Z(\phi)$ in series of basis functions. Let $P_{i}(\phi)$ and $Q_{i}(\phi)$ designate elements of sets of basis functions for $Y(\phi)$ and $Z(\phi)$, respcctively, such that

$$
Y(\phi)=\sum_{i=1}^{M} c_{i} P_{i}(\phi)
$$

and

$$
Z(\phi)=\sum_{i=1}^{M} c_{i} Q_{i}(\phi)
$$

For simplicity of implementation, we take the same number of basis functions in both regions. When equation (15) is projected over the basis functions $P_{j}(\phi)$ and equation (16) over $Q_{j}(\phi)$, we get two sets of lincar and homogeneous equations in the coefficients $c_{i}$ and $d_{i}$, namely

$$
\begin{aligned}
& {[A][c]+[B][d]=0} \\
& {[C][c]+[D][d]=0}
\end{aligned}
$$

The entries of the matrices in equations (18) are given by

$$
\begin{gathered}
{[A]_{i j}=\sum_{n=0}^{\infty} \frac{J_{n}^{\prime}\left(k_{c} b\right)}{J_{n}\left(k_{c} b\right)} \tilde{P}_{i}^{I c}(n) \tilde{P}_{j}^{I c}(n)\left(1+\delta_{n 0}\right)-\left(1-\frac{\theta}{\pi}\right) \times} \\
\sum_{m=0}^{\infty} \frac{J_{l}^{\prime}\left(k_{c} b\right) Y_{l}^{\prime}\left(k_{c} a\right)-J_{l}^{\prime}\left(k_{c} a\right) Y_{l}^{\prime}\left(k_{c} b\right)}{J_{l}\left(k_{c} a\right) Y_{l}^{\prime}\left(k_{c} a\right)-Y_{l}\left(k_{c} b\right) J_{l}^{\prime}\left(k_{c} a\right)} \tilde{P}_{i}^{I I c}(m) \tilde{P}_{j}^{I I c}(m)\left(1+\delta_{m 0}\right) \\
{[B]_{i j}=\sum_{n=0}^{\infty} \frac{J_{n}^{\prime}\left(k_{c} b\right)}{J_{n}\left(k_{c} b\right)} \tilde{P}_{i}^{I c}(n) \tilde{Q}_{j}^{I c}(n)\left(1+\delta_{n 0}\right)} \\
{[C]_{i j}=\sum_{n=0}^{\infty} \frac{J_{n}^{\prime}\left(k_{c} b\right)}{J_{n}\left(k_{c} b\right)} \tilde{Q}_{i}^{I c}(n) \tilde{P}_{j}^{I c}(n)\left(1+\delta_{n 0}\right)=[B]_{j i}}
\end{gathered}
$$

and

$$
[D]_{i j}=\sum_{n=0}^{\infty} \frac{J_{n}^{\prime}\left(k_{c} b\right)}{J_{n}\left(k_{c} b\right)} \tilde{Q}_{i}^{I c}(n) \tilde{Q}_{j}^{I c}(n)\left(1+\delta_{n 0}\right)
$$

The cutoff wavenumbers are again determined as the roots of the determinant of the extended matrix whose submatrices are given by (19).

\section{B BASIS FUNCTIONS FOR H-FORMULATION}

The basis functions used to expand $H_{z}$ in each of the two intervals of the interface I-II should contain the edge conditions to accelerate numerical 
convergence. Since $H_{z}$ is parallel to the axis of the $90^{\circ}$ metallic wedge, it becomes non-analytic as $r^{2 / 3}$ as the radial distance to the axis of the wedge $r$ approaches zero [7]. However, the function $J_{0}(\gamma r)$, which approaches a constant as $r \rightarrow 0$, is also an asymptotic solution for the TE modes of a metallic wedge of non-zero internal angle [7]. This function dominates the axial magnetic field in the vicinity of the edges of the ridge. Taking this important point into consideration, we use the following basis functions

$$
P_{k}(\phi)=\cos \left[k \pi \frac{\phi-\theta}{\pi-\theta}\right], \quad k=0,1,2, \ldots
$$

and

$$
Q_{k}(\phi)=\cos \left[k \pi \frac{\phi}{\theta}\right], \quad k=0,1,2, \ldots
$$

These basis functions emphasize the contribution of the constant asymptotic solution. Their spectra are given by the expressions

$$
\begin{aligned}
& \tilde{P}_{k}^{I c}(n)= \begin{cases}1-\frac{\theta}{\pi}, & n=k=0 \\
\frac{-2 n \sin (n \theta)}{\pi\left[n^{2}-\left(\frac{k}{1-\frac{l}{\pi}}\right)^{2}\right]}, & n \neq 0\end{cases} \\
& \tilde{P}_{k}^{I I c}(m)=\delta_{k m}
\end{aligned}
$$

and

$$
\tilde{Q}_{k}^{I c}(n)=\frac{0}{\pi} \frac{1}{1+\delta_{n 0}}\left[\frac{\sin (n \theta+k \pi)}{n \theta+k \pi}+\frac{\sin (n \theta-k \pi)}{n \theta-k \pi}\right]
$$

Other basis functions which behave as $r^{2 / 3}$ in the vicinity of the ridges were also tried and failed to predict the correct cutoff wavenumbers.

Note that the modes of the two subregions are again all included in the theory and rather in a simple way; they appear only in computing the inner products in the entries of the submatrices of (19). The phenomenon of relative convergence is eliminated by testing these sums for convergence thereby leaving only one free parameter in the numerical solution, i.e., the number of basis functions $M$.

The spectra of the basis functions of the TE modes with magnetic-wall symmetry are given in Appendix B.

\section{NUMERICAL RESULTS AND DISCUSSION}

The cutoff wavenumbers of the first few TE modes of the ridge circular waveguide shown in figure 1 were determined using both formulations. 


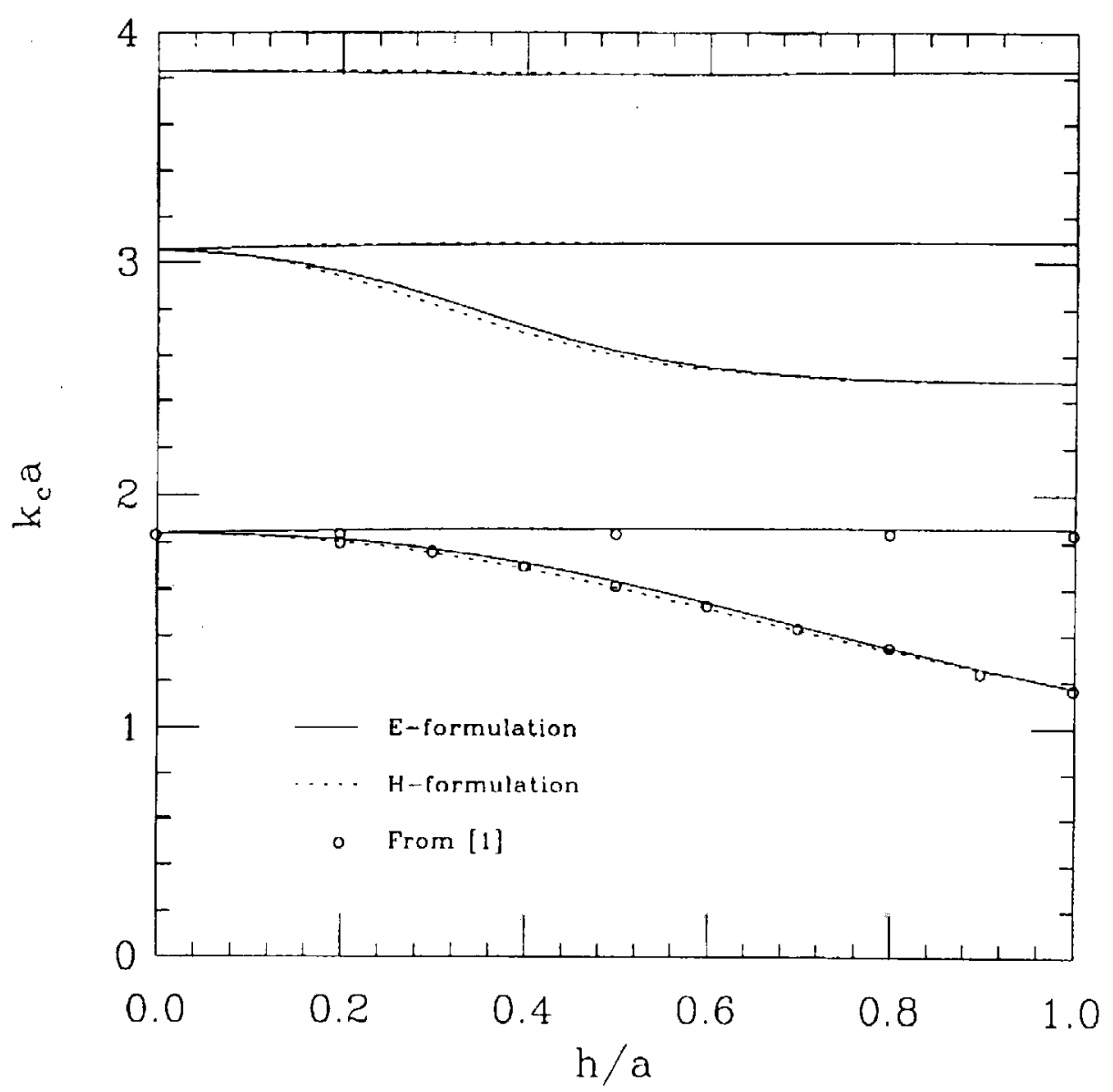

Figure 2. Cutoff wavenumbers of the TE modes as a function of $h / a$ with $\theta=2.292^{\circ}$ from E-formulation (solid line) and $\mathrm{H}$-formulation (dashed line). The circles are from reference [1].

The first structure we investigated is a narrow ridge, $\theta=2.292^{\circ}$. Figure 2 shows the cutoff wavenumbers of the first $3 \mathrm{TE}$ modes with electric wall symmetry as a function of the depth of the ridge. The E-formulation converges with 4 basis functions whereas up to 6 basis functions are necessary for the $\mathrm{H}$-formulation to converge. The circles show the excellent agreement between the results of this work and the finite element calculation presented in [1]. An additional test of our numerical results is provided by the cutoff 
wavenumbers of the sectoral waveguide which are given by the equation

$$
J_{l}^{\prime}\left(k_{c} a\right)=0, \quad l=\frac{m}{1-\frac{\theta}{\pi}}, \quad m=0,1,2, \ldots
$$

for the TE modes with an electric wall symmetry. For the TE modes with magnetic wall symmetry, the cutoff wavenumbers are given by the same equation but with $l=\frac{m-1 / 2}{1-\frac{\theta}{\pi}}$. Results from both formulations converge in the limit $h \rightarrow 0$, to those given by equation (22). It is also interesting to note that the cutoff wavenumber of the $\mathrm{TE}_{01}$ mode $\left(k_{c} a=3.832\right)$ is the same for both the empty and the sectoral waveguide. This is also reproduced by both formulations.

In computing the sums in equations (10) for the E-formulation, we found that 30 terms were sufficient to guarantec convergence. On the other hand, the sums in equations (19) in the H-formulation require more than 100 terms to obtain numerically stable results. The reason resides in the fact that the spectrum of the basis functions $\tilde{Q}_{i}^{I c}(n)$ have a long tail because of the small width of the ridge. It is, however, important to note that more terms are needed when a larger number of basis functions are used. This is the case when the cutoff frequencies of higher order modes are required such as in computing generalized scattering matrices of an empty to ridged waveguide transition.

The second structure is a wider ridge, $0=11^{\circ}$, for which both formulations converge with 4 basis functions but the sums in equations (19) still require approximately 80 terms to converge whereas those in equation (10) converge with 30 terms. If a much wider ridge is assumed, $0=45^{\circ}$ for example, the sums in the $\mathrm{H}$-formulation converge with only 30 terms. Figure 3 shows the cutoff wavenumbers of the first $3 \mathrm{TE}$ modes with an electric wall symmetry and the first 2 TE modes with a magnetic wall symmetry as a function of the ridge depth $h / a$. Both formulations converge to the same results and reproduce the cutoff wavenumbers of the empty and sectoral waveguides.

From the present numcrical results, and taking into account the fact that the E-formulation suffers from spurious roots which are, nevertheless, eliminated when enough basis functions are used, the H-formulation is efficient in analyzing wide ridges, whereas the E-formulation should be adopted for narrow ridges. A combination of both formulations may also be advantagcous for narrow ridges where the results of the H-formulation, which docs not give spurious roots, are used as starting guesses for the F-formulation. The Hformulation requires roughly twice as much CPU time as the E-formulation. 


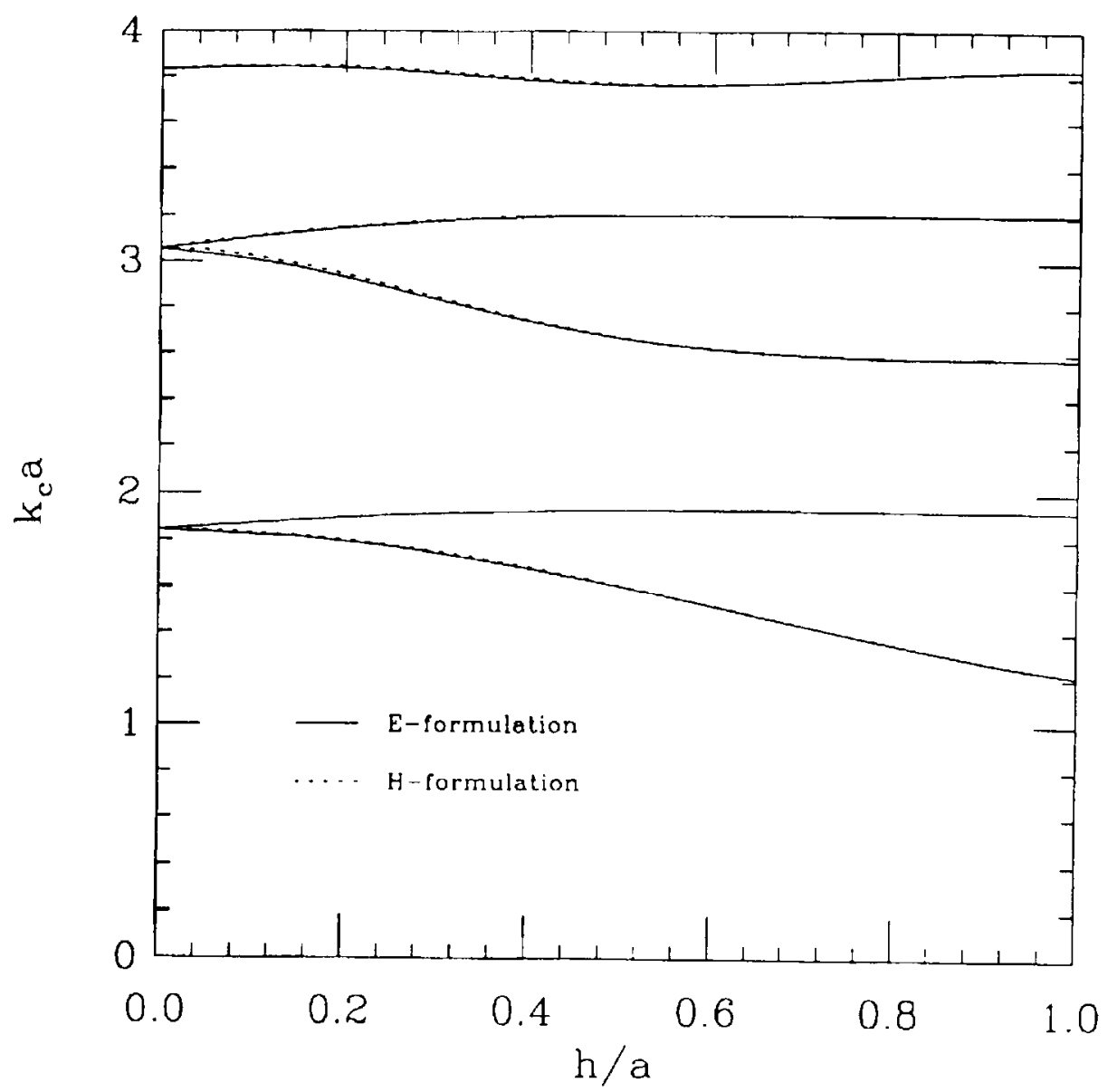

Figure 3. Cutoff wavenumbers of the TE modes as a function of $h / a$ with $\theta=11^{\circ}$ from E-formulation (solid line) and II-formulation (dashed line).

\section{CONCLUSIONS}

A comparative study of two integral-equation formulations of the TE cutoff wavenumbers in a ridge circular waveguide was presented. The E-formulation consists in deriving and solving an integral equation for $E_{\phi}$ and was found to suffer from spurious roots which are eliminated when enough basis functions are used. On the other hand, the H-formulation in which an integral equation for the axial magnetic field $H_{z}$ is solved requires larger matrices but does not exhibit any spurious roots. The H-formulation is effective in analyzing wide ridges $\left(0 \geq 10^{\circ}\right)$ whereas the E-formulation is 
appropriate for narrow ridges. A combination of both formulations allows an efficient analysis of ridges of arbitrary width.

\section{APPENDIX A}

In this appendix we give the essential formulas for the TE modes with magnetic wall symmetry within the E-formulation.

The entries of the matrix which corresponds to equation (10) are given by the following equation

$$
\begin{aligned}
{[K]_{i j}=\left(1-\frac{\theta}{\pi}\right) } & \sum_{m=1}^{\infty} \tilde{B}_{i}^{I I c}(m) \tilde{B}_{j}^{I I c}(m) \times \frac{Y_{l}^{\prime}\left(k_{c} a\right) J_{l}\left(k_{c} b\right)-J_{l}^{\prime}\left(k_{c} a\right) Y_{l}\left(k_{c} b\right)}{Y_{l}^{\prime}\left(k_{c} a\right) J_{l}^{\prime}\left(k_{c} b\right)-J_{l}^{\prime}\left(k_{c} a\right) Y_{l}^{\prime}\left(k_{c} b\right)} \\
& -\sum_{n=1}^{\infty} \frac{J_{n}\left(k_{c} b\right)}{J_{n}^{\prime}\left(k_{c} b\right)} \tilde{B}_{i}^{I s}(n) \tilde{B}_{j}^{I s}(n)
\end{aligned}
$$

where $l=\frac{m-1 / 2}{1-\theta / \pi}$.

The basis functions for these modes are given by

$$
B_{k}(\phi)=\frac{\cos \left[(2 k-1) \frac{\pi}{2} \frac{\phi-\theta}{\pi-\theta}\right]}{[(\phi-\theta)(2 \pi-\theta-\phi)]^{1 / 3}}, \quad k=1,2,3, \ldots
$$

The spectra of these basis functions are defined and given by

$$
\begin{aligned}
\tilde{B}_{k}^{I s}(m)= & \frac{1}{\pi} \int_{0}^{2 \pi-\theta} \sin (m \phi) \frac{\cos \left[(2 k-1) \frac{\pi}{2} \frac{\phi-\theta}{\pi-0}\right]}{[(\phi-0)(2 \pi-0-\phi)]^{1 / 3}} d \phi \\
= & -G(-1)^{m+k}\left[\frac{J_{1 / 6}\left[\left(m+\frac{2 k-1}{2}\right) \pi-m \theta\right]}{\left|\left(m+\frac{2 k-1}{2}\right) \frac{\pi}{2}-m \frac{\theta}{2}\right|^{1 / 6}}\right. \\
& \left.-\frac{\left.J_{1 / 6} \|\left(m-\frac{2 k-1}{2}\right) \pi-m 0 \mid\right]}{\left|\left(m-\frac{2 k-1}{2}\right) \frac{\pi}{2}-m \frac{0}{2}\right|^{1 / 6}}\right]
\end{aligned}
$$

and

$$
\begin{aligned}
\tilde{B}_{k}^{I I c}(m) & =\frac{1}{\pi-\theta} \int_{0}^{2 \pi-\theta} \cos \left[(2 m-1) \frac{\pi}{2} \frac{\phi-0}{\pi-0}\right] \frac{\cos \left[(2 k-1) \frac{\pi}{2} \frac{\phi-\theta}{\pi-\theta}\right]}{[(\phi-\theta)(2 \pi-0-\phi)]^{1 / 3}} d \phi \\
& =-G(-1)^{m+k}\left[\frac{J_{1 / 6}[(m+k-1) \pi]}{\left|(m+k-1) \frac{\pi}{2}\right|^{1 / 6}}-\frac{J_{1 / 6}[|(m-k) \pi|]}{\left.|m-k| \frac{\pi}{2}\right|^{1 / 6}}\right]
\end{aligned}
$$

\section{APPENDIX B}

In this appendix we summarize the formulae for the TE modes with magnetic-wall symmetry within the H-formulation. 
The entries of the matrices corresponding to equations (19) are given by

$$
\begin{aligned}
{[A]_{i j} } & =\sum_{n=1}^{\infty} \frac{J_{n}^{\prime}\left(k_{c} b\right)}{J_{n}\left(k_{c} b\right)} \tilde{P}_{i}^{I s}(n) \tilde{P}_{j}^{I s}(n) \\
& -\left(1-\frac{\theta}{\pi}\right) \sum_{m=1}^{\infty} \frac{J_{l}^{\prime}\left(k_{c} b\right) Y_{l}^{\prime}\left(k_{c} a\right)-J_{l}^{\prime}\left(k_{c} a\right) Y_{l}^{\prime}\left(k_{c} b\right)}{J_{l}\left(k_{c} a\right) Y_{l}^{\prime}\left(k_{c} a\right)-Y_{l}\left(k_{c} b\right) J_{l}^{\prime}\left(k_{c} a\right)} \tilde{P}_{i}^{I I c}(m) \tilde{P}_{j}^{I I c}(m)
\end{aligned}
$$

where $l=\frac{m-1 / 2}{1-\theta / \pi}$.

$$
\begin{aligned}
& {[B]_{i j}=\sum_{n=1}^{\infty} \frac{J_{n}^{\prime}\left(k_{c} b\right)}{J_{n}\left(k_{c} b\right)} \tilde{P}_{i}^{I s}(n) \tilde{Q}_{j}^{I s}(n)} \\
& {[C]_{i j}=\sum_{n=1}^{\infty} \frac{J_{n}^{\prime}\left(k_{c} b\right)}{J_{n}\left(k_{c} b\right)} \tilde{Q}_{i}^{I s}(n) \tilde{P}_{j}^{I s}(n)}
\end{aligned}
$$

and

$$
[D]_{i j}=\sum_{n=1}^{\infty} \frac{J_{n}^{\prime}\left(k_{c} b\right)}{J_{n}\left(k_{c} b\right)} \tilde{Q}_{i}^{I s}(n) \tilde{Q}_{j}^{I s}(n)=[B]_{j i}
$$

The following basis functions where used

$$
P_{k}(\phi)=\cos \left[(2 k-1) \frac{\pi}{2} \frac{\phi-\theta}{\pi-0}\right], \quad 0 \leq \phi \leq 2 \pi-\phi, \quad k=1,2, \ldots
$$

and

$$
Q_{k}(\phi)=\sin \left[(2 k-1) \frac{\pi}{2} \frac{\phi}{0}\right], \quad-0 \leq \phi \leq 0, \quad k=1,2, \ldots
$$

The spectra of these basis functions are given by

$$
\begin{gathered}
\tilde{P}_{k}^{I s}(n)=-\frac{2}{\pi} \frac{n \cos [n \emptyset]}{n^{2}-\left(\frac{2 k-1}{2(1-0 / \pi)}\right)^{2}} \\
\tilde{P}_{k}^{I I c}(m)=\delta_{m k}
\end{gathered}
$$

and

$$
\tilde{Q}_{k}^{I s}(n)=-\frac{2}{\pi} \sin \left[\frac{2 k-1}{2} \pi\right] \frac{n \cos [n \varrho]}{n^{2}-\left(\frac{2 k-1}{20 / \pi}\right)^{2}}
$$




\section{REFERENCES}

1. Behe. R., and P. Brachat, "Compact duplexer-polarizer with semicircular waveguide," IEEE Trans. Antennas Propagat., Vol. 39, 12221224, Aug. 1991.

2. Balaji, U., and R. Vahldieck, "Radial mode matching analysis of ridged circular waveguide," in 1995 IEEE MTT-S Int. Microwave Symp. Dig., $637-640$.

3. Xiao, S., R. Vahldieck, and M. Gugliemi, "Field theory analysis of circular ridge waveguides with partial dielectric filling," in 1995 IEEE MTT-S Int. Microwave Symp. Dig., 265-268.

4. Sun, W., and C. A. Balanis, "Analysis and design of quadruple ridged waveguides," IEEE Trans. Microwave Theory Tech., Vol. 42, 22012207 , Dec. 1994.

5. Harrington, R. F., Field Computation by the Moment Methods, Krieger, Malabar, FL., 1987.

6. Labay, V. A., and J. Bornemann, "Matrix singular value decomposition for pole-free solutions of homogeneous matrix equations as applied to numerical modeling methods," IEEE Microwave and Guided Wave Letl., Vol. 2, No. 2, 49-51, Feb. 1992.

7. Colllin, R. E., Field Theory of Guided Waves, IFFF, Press, New York 1991.

8. Gradshteyn, I. S., and I. M. Ryznik, Tables of Integrals, Series, and Products, Fifth Edition, Academic Press, New York, 1994.

Smain Amari received his DFS in physics and electronics from Constantine University (Algeria) in 1985, the M.S. degree in electrical engincering in 1989 and the Ph.D. degree in physics in 1994 both from Washington University in St. Louis. He is interested in numerical methods in electromagnetics, numerical analysis, applied mathematics, applicd physics and application of quantum field theory in quantum many-particle systerns. Since 1994, he has been with the Department of Elcctrical and Computer Enginecring at the University of Victoria, Canada.

Jens Bornemann received the Dipl.-Ing. and Dr.-Ing. degrees in clectrical engineering from the University of Bremen, Germany, in 1980 and 1984, respectively. He is currently a Professor in the Department of ECE, University of Victoria, B.C., Canada. IIs research activities focus on microwave components design, and electromagnetic field theory in circuits and antennas. Dr. Bornemann is a Senior Member of IEEE and serves on the editorial boards of IEEE Trans. MTT and Inl. J. of Numerical Modelling. He has (co)authored more than 100 technical papers and a book on Waveguide Components for Antenna Feed Systems - Theory and CAD, Artcch House, 1993. 
Rüdiger Vahldieck (M'85-SM'86) received the Dipl.-Ing. and the Dr.-Ing. degrees in electrical engineering from the University of Bremen, West Germany, in 1980 and 1983, respcctively. From 1984 to 1986 he was a Research Associate at the University of Ottawa, Canada. In 1986 he joined the University of Victoria, British Columbia, Canada, where he became a Full-Professor in the Department of Electrical and Computer Fngineering in 1991. During Fall and Spring 1992-93 he was a visiting scientist at the "Ferdinand-Braun-Institute für Hochfrequenztechnik" in Berlin, Germany. Since October 1996 he has been the Chair for Field Theory at the Laboratory for Electromagnetic Fields and Microwave Electronics at the Swiss Federal Institute of Technology in Zurich, Switzerland. His rescarch interests include numerical methods to model electromagnetic fields for computer-aided design of microwave, millimeter wave and opto-electronic integrated circuits. $\mathrm{He}$ is also interested in the design and simulation of devices and subsystems for broadband fiber-optic communication systcms. Dr. Vahldieck, together with three coauthors, received the outstanding publication award of the Institution of Electronic and Radio Engincers in 1983. Ile is on the cditorial board of the IEEE Transaction on Microwave Theory and Techniques and since 1992 serves on the Technical Program Committee of the IEEE International Microwave Symposium. He has published more than 150 technical papers. 